\title{
A Half-Bridge HTS Transformer-Rectifier Flux Pump with two AC field-controlled switches
}

\author{
J.D.D Gawith, J. Geng, C. Li, B. Shen, X. Zhang, J. Ma, T.A. Coombs, \\ University of Cambridge \\ 9 JJ Thomson Ave \\ Cambridge CB3 OFA \\ United Kingdom
}

\begin{abstract}
Flux pumping provides a method of inductively magnetizing superconducting coils and can provide thermal, electrical and mechanical isolation between cryogenic environment and power supply. This offers the possibility of lower cryogenic loading and more flexible arrangements for superconducting magnet systems. This paper reports on the performance and analysis of an HTS half-bridge transformer-rectifier type flux pump using two switching elements formed by applying AC magnetic field to sections of HTS. Theory of operation is presented followed by results from an experimental prototype which are compared with SPICE simulation. There is good qualitative agreement between simulation and experiment and all current, voltage and power waveforms from experiment are presented. This work represents a step forward in the understanding of the operation of HTS transformer-rectifier flux pumps and shows that SPICE simulation can provide designers of future HTS flux pumping systems with a useful tool for making design decisions.
\end{abstract}

Contact: jddg2@ cam.ac.uk, jg717@cam.ac.uk

Index Terms -Flux Pump, Superconductor, HTS, Transformer-Rectifier, Half-Bridge

\section{Introduction}

Recently there has been increasing interest in using REBCO magnets for applications requiring high magnetic field density including NMR [1], particle accelerators [2] and plasma confinement [3]. There is also interest where the high current density and low loss of these materials at practical temperatures can provide advantages over existing systems such as for superconducting electrical machines which can be made smaller and lighter for the same power and torque specification [4].

One of the main drawbacks in using superconducting magnets rather than conventional permanent magnets is that the former require dedicated power supplies to inject and remove magnetic flux. Typically these are straightforward power supplies which rectify power from the grid then have a DC-DC conversion stage to lower the voltage and boost the current [5]. Flux pumps are an alternative means of energizing superconducting magnets which eliminates the need for current leads between the magnet and the external environment. Removal of current leads has the potential to reduce system cooling requirements due to the elimination of the thermal link between the magnet at cryogenic temperature and the power supply at ambient temperature. It also allows for physical separation between magnet and power supply which provides more flexible arrangements which can be advantageous in some applications like rotating machines.

There has been much recent work detailing the physical mechanisms behind HTS flux pumps [6][7][8], the theory of operation of HTS flux pumps [9][10] and results from different
HTS flux pumps operating under various conditions [9][10][11][12]. There is an identified need in the field of HTS flux pumping for a bridging of the gap between basic physical theory and operational performance. This paper presents a detailed model and simulations of an HTS flux pump based on physical principles which can predict operational performance including maximum output current, charging voltage and power losses in the device.

An HTS Transformer-Rectifier Flux Pump (TRFP) switched by an AC field was proposed by Geng et al [7], which uses only one switching element. The work in this paper focuses on an HTS TRFP which uses two AC field HTS switching elements arranged in a half-bridge configuration. A short review of flux pumps is given followed by the theory of operation of TRFPs. This theory of operation is used to develop a simplified circuit model using SPICE software and the operational behavior of the half-bridge TRFP is explored. Based on this modelling a prototype flux pump was built and its performance compared to simulation. Importantly this is the first time an analysis of all voltage, current and power waveforms has been given for an HTS flux pump. Conclusions are drawn about the accuracy of modelling and the ramifications of this work for the construction and testing of future flux pumps.

\section{Flux Pumping Theory}

Flux pumps have been designed and built for many decades [13] with designers taking several different routes but all relying on the same fundamental physics of magnetic induction to induce an $\mathrm{AC}$ voltage and time variable impedance to rectify this voltage. 
While there is current work on many types of HTS flux pump such as the DC dynamo pump [14] [10] and the linear type flux pump [15] [16] [17], the work in this paper focuses on the transformer-rectifier flux pump (TRFP). In their review of Low Temperature Superconducting (LTS) flux pumps, van der Klundert and Ten Kate [13] compared the fundamental working principles and governing equations for various types of flux pump. They concluded that the transformer-rectifier gives the designer more flexibility due to its separation of the source of the EMF (the transformer) and the method of varying the impedance (the rectifier circuit).

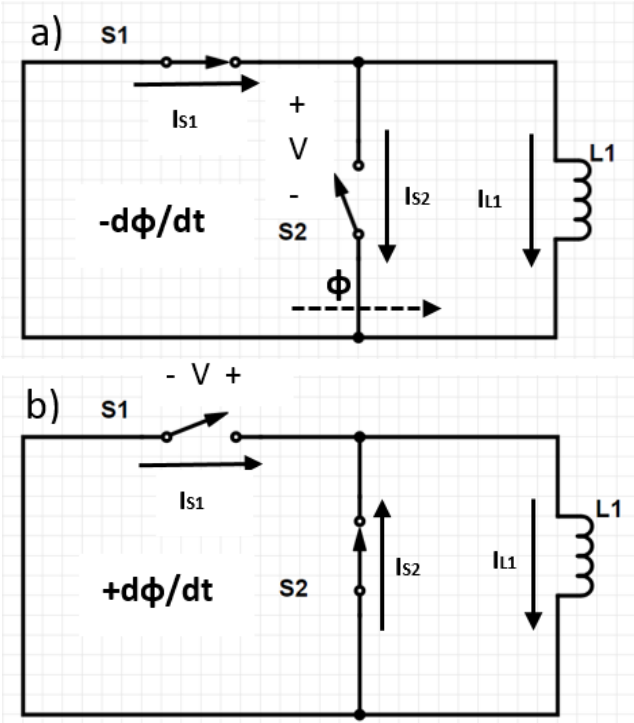

Figure 1: Flux pump equivalent circuit model. a) first half cycle $S 1$ is low impedance and S2 is high impedance, allowing flux to transfer from Loop 1 to Loop 2 which is associated with a voltage across S2. b) second half-cycle $\mathrm{S} 1$ is high impedance and S2 is low impedance. The emf produced is dropped across $\mathrm{S} 1$ and the current flowing in the load is commutated to flow through S2. Over a full cycle, the load sees a voltage in only one sense and will gradually accumulate current with repeated cycles.

Figure 1 shows the basic flux pump model. Flux pumps can be considered as a two-loop system with two switching elements, S1 and S2. In this case, the switches do not necessarily have to be ideal, one or both must simply provide a mechanism to vary impedance over time. If the left-hand loop experiences a time changing, periodic, $\mathrm{AC}$ magnetic field $d \Phi / d t$, from Faraday's law an EMF will be induced around the loop. If the impedance of S1 or S2 are modulated in phase with the AC flux through the left-hand loop, it is possible to have a time-averaged DC voltage across the load coil L1. Geng et al [18] recently formalised the value of this DC voltage across the load as:

$$
V_{D C}=\frac{1}{T} \int_{0}^{T} \frac{-\frac{d \Phi}{d t}(t)}{R_{S 1}(t)+R_{S 2}(t)} R_{S 2}(t) d t
$$

Note that this equation is only valid when the load current, $\mathrm{I}_{\mathrm{L} 1}=0$, i.e. at the start of pumping. Assuming flux per cycle and its time derivative integrate to zero, one or both resistances $R_{S 1}(t)$ and $R_{S 2}(t)$ must vary over time to produce a DC voltage.

In previous HTS transformer-rectifier flux pumps [7] [19] [20], only $R_{\mathrm{S} 2}(t)$ has been varied over time. In the case of the single-switch pump, $R_{S 2}(t)$ is time variant and $R_{S 1}$ is largely ignored. However, if $R_{S 1}=0$ then flux pumping cannot be achieved, as seen from Eq. (1). This is because $R_{\mathrm{S} 2}(t)$ is present and equal on both the numerator and denominator so cancel out to 1 . The only term left within the integral is $\frac{-d \Phi}{d t}$ which we have already stated will integrate to zero over a full period. In the absence of any $R_{S 1}$, flux pumping cannot theoretically occur. However, it is important to note that there are always small fixed resistances present in the circuit due to joints and AC loss mechanisms which allow for pumping even in the absence of a dedicated $R_{S 1}$. This explains the results in previous HTS flux pumps that have not included a dedicated $\mathrm{R}_{\mathrm{S} 1}$.

Consider the case where $\mathrm{R}_{\mathrm{S1}}$ is large. From equation 1, the DC voltage will be small as the denominator of the integral will be very large. This is clearly not the optimal case for maximizing DC voltage across the load.

Now take the case where $R_{S 1}$ is very small. The voltage produced by changing flux in the loop splits between $\mathrm{S} 1$ and $\mathrm{S} 2$. If the resistance of $\mathrm{S} 2$ is no-zero, most of the voltage will drop across S2. This will happen in both the positive and negative half-cycles, resulting in an integral voltage across S2 which is actually quite small. The limiting case of this is where $\mathrm{R}_{\mathrm{S} 1}=0$ and there is no voltage across the load.

Allowing $\mathrm{S} 1$ to become a dynamic $\mathrm{R}_{\mathrm{S} 1}(\mathrm{t})$ or switching element means that it can present a low impedance in the first half cycle (all voltage dropped across S2), then it can present a high impedance in second half cycle (all voltage dropped across $\mathrm{S} 1)$. With $\mathrm{R}_{\mathrm{S} 2}(\mathrm{t})$ switching 180 degrees out of phase this forms a half bridge rectification circuit which is the reason this topology is used instead of a single switch configuration. These relationships and the importance on the value of $R_{S 1}$ are explored through simulation and experimentation and in section VI.

\section{SPICE Simulation of Transformer Rectifier Flux Pump}

The literature from sections I and II agree that flux pumps can be modelled as simplified circuits [6][8]. A natural extension of this idea is to model and analyze the circuit in SPICE simulation software. The simulations seen throughout this paper are in the program LtSPICE and are based on the circuit shown in Figure 2. These simulations help with understanding the operation of the system including waveform shape and power transfer. This simulation analysis and the comparison with laboratory results in section VI signify a step forward in the understanding of HTS flux pumps. 


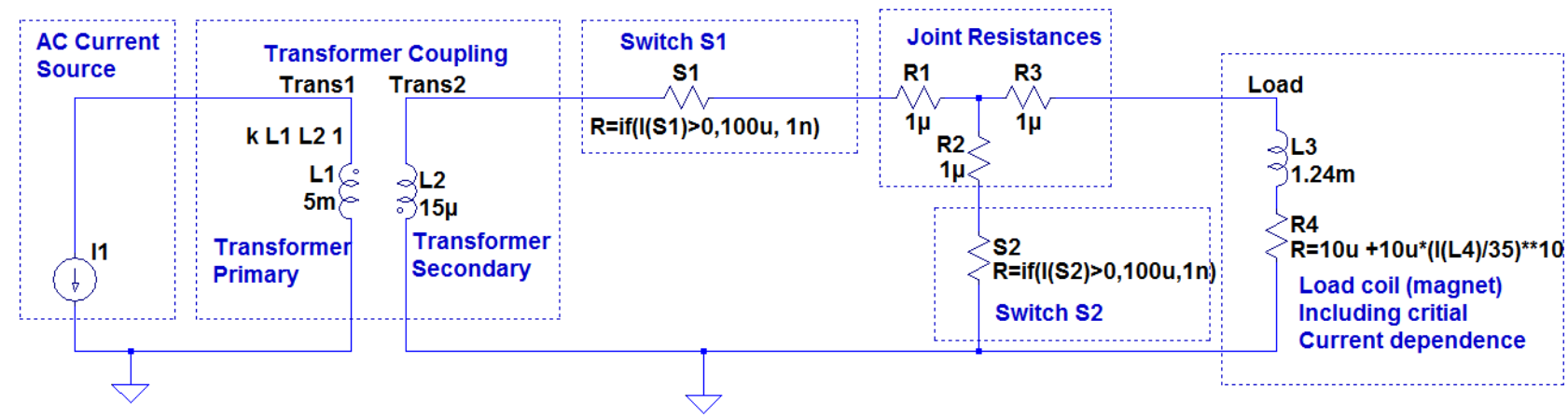

Figure 2: SPICE simulation of transformer rectifier flux pump. A trapezoidal current I1 flows though transformer primary L1, which induces a voltage across transformer secondary L2. This AC voltage is partially rectified by HTS switching elements S1 and S2 and a DC voltage component is seen across the load $\mathrm{L} 3$, which has an inductance of $1.24 \mathrm{mH}$. R1, R2 and R3 are joint resistance all modelled as $1 \mu \Omega$ and $\mathrm{R} 4$ is the non-linear load resistance which models an HTS tape with a critical current of 35A and an $n$ value of 10 . The code specifying the behaviour of $\mathrm{S} 1$ and S2 means that the impedance of S1 is varied synchronously with primary transformer current between $100 \mu \Omega$ and $1 \mathrm{n} \Omega$, just like in the experiment. The value of $1 \mathrm{n} \Omega$ is meant to replicate the superconducting state when there is no applied field in the switch.

\section{AC Magnetic Field Switches}

The choice of switching element for a flux pumping system is important. In a traditional rectifier circuit, switches are semiconductor-based devices such as MOSFETs and IGBTs. These devices provide a very high off-state resistance $(\approx \mathrm{M} \Omega \mathrm{s})$, have low on-state resistance $(\approx \mathrm{m} \Omega \mathrm{s})$ and have fast switching between states $(\approx n s)$. While transformer-rectifier flux pumps have been built using these devices [21], their on-state resistance means that they cannot operate in persistent mode and so need a constantly operating power supply. Typically flux pumps have used superconducting switching elements which have no resistance in the on-state and non-zero resistance in the off-state. The two major mechanisms for developing resistance in these elements has been to heat a section of superconductor above $T_{C}$ to form a thermal switch or exposing a section of superconductor to a field greater than $\mathrm{B}_{\mathrm{C}}$ forming a magnetic switch. While this was practical for LTS devices, in HTS devices the material thermal conductivity is low so heating above Tc would lead to a long recovery time and due to HTS's high upper critical field, driving the material above $\mathrm{B}_{\mathrm{C}}$ would be also be impractical.

Geng et al [22] recently proposed an HTS switching element which uses an AC magnetic field applied to a length of HTS to generate a dynamic resistance. The field can be much lower than $\mathrm{B}_{\mathrm{C}}$ and does not need to heat the HTS to produce a resistance. Geng et al [7] used this switching element to create a flux pumping system.

The mechanism for these switches was identified by Andrianov et al [24] who showed that a type II superconductor carrying a DC transport current will generate a DC voltage when a perpendicular AC magnetic field is applied to it. This analysis was extended by Jiang et al [25] giving the following equation for dynamic resistance.

$$
R_{d \perp}=\frac{4 a L f}{I_{c 0}}\left(B_{a, \perp}-B_{t h, \perp}\right) \text { for } \mathrm{B}_{\mathrm{a}, \perp} \gg \mathrm{B}_{\mathrm{th} \perp}
$$

where $a$ is the half width of the slab, $\mathrm{L}$ is the length of the slab subjected to the field, $\mathrm{f}$ is the field frequency, $\mathrm{I}_{\mathrm{C} 0}$ is the critical current of the slab, $B_{a, \perp}$ is the magnitude of the field applied perpendicular to the tape, and $B_{t h, \perp}$ is the threshold field given in Ref. [25].
Seen from this equation, to develop a higher resistance, the frequency or field strength can be increased. The field strength is typically limited by core material selection while frequency is limited by core material and power supply requirements. One way to extend the frequency range is to capacitively compensate the electromagnet so the power supply is driving a lower impedance. At higher frequency, it is desirable to use suitable core material such as ferrite. With this in mind, the two switching elements used in this prototype were made using ferrite cores, capacitively tuned to and driven at $2.5 \mathrm{kHz}$. One of these switches can be seen in Figure 3.

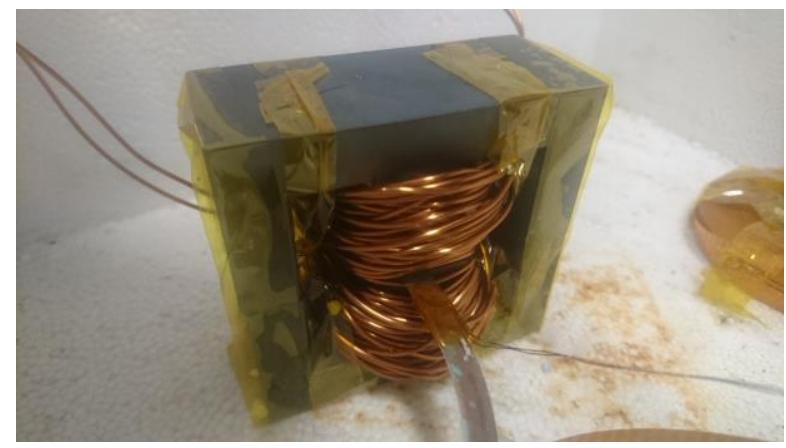

Figure 3: One of the two AC field superconducting switching elements used in the prototype flux pump. A copper coil is wound around the centre of a ferrite double E-core that has a $2 \mathrm{~mm}$ gap in the centre for the superconductor to pass through. The copper coil is wound bifilar to reduce its impedance so that it can be driven at $2.5 \mathrm{kHz}$. The superconducting strip is soldered at the far end to form a non-inductive section which the field is applied to.

\section{Experimental System and Setup}

The prototype setup formed a circuit equivalent to the simulated circuit shown in Figure 2. For the prototype, the AC current source I1 is a KEPCO power supply driving a $1 \mathrm{~Hz}, 6 \mathrm{~A}$ peak trapezoidal current into the primary side of a 100:3 stepdown transformer. The secondary of the transformer connects to two AC field superconducting switching elements, which together form a half-bridge rectifier. This is then connected to a load magnet.

The system is controlled by a program written in LabVIEW which consists of driving three synchronously controlled 
analogue output waveforms, one for the Kepco power supply and two for the EP4000 power supply. All driving signals were created, and measurements taken using a NI 6323 data acquisition card which can sample at $250 \mathrm{kHz}$, has 4 analogue outputs and 32 analogue inputs.

\section{Results}

The first result to obtain was the dynamic resistance of the switching elements in the off-state. Figure 4 shows the average current vs average voltage for $\mathrm{S} 1$. The data has been averaged over 100 samples to show only the time-averaged effect of dynamic resistance and dynamic voltage for clarity, as well as to reduce noise. The average dynamic resistance is calculated taking the average dynamic voltage divided by average current only when the voltage is significant $\left(\left|V_{s 1}\right|>1 \mathrm{mV}\right)$. Figure 5 shows the average voltage and resulting average resistance when $\left|V_{s 1}\right|>1 \mathrm{mV}$, giving an off-state average value of around $100 \mu \Omega$. This value does have some variation both during each cycle and as the load current changes, however it is a useful measure and using this averaged value gives good agreement with simulation.

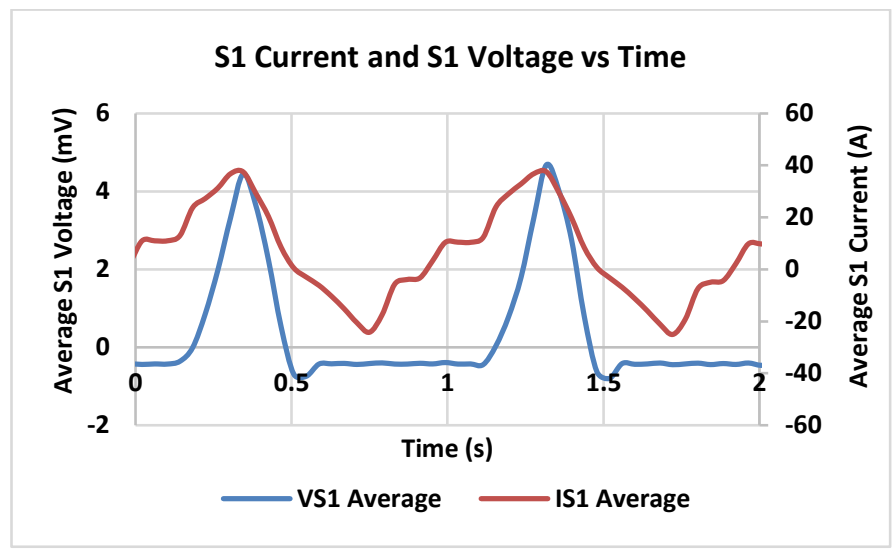

Figure 4: The current through switching element S1 and the voltage across it over two full cycles.

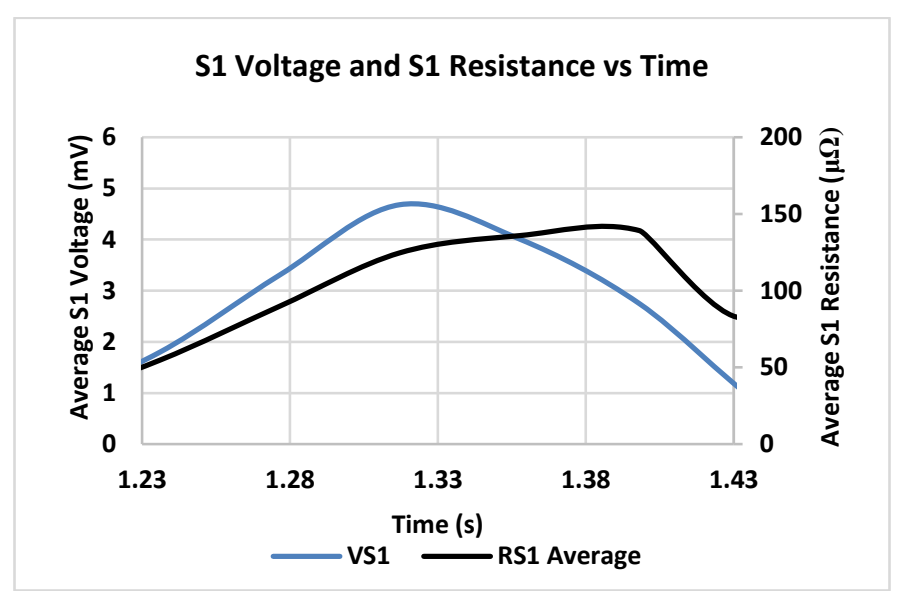

Figure 5: The dynamic voltage and dynamic resistance of switch S1 for the part of the cycle where there is an appreciable voltage across it.

Once it had been established that the switching elements were working, the next step was to compare the performance of a single-switch flux pump, with a static S1 to a two-switch pump with a switched S1. Figure 8 shows a SPICE simulation with four curves. The first three show simulations where $S 1$ is just a fixed resistance $(1 \mu \Omega, 10 \mu \Omega, 100 \mu \Omega)$. The fourth (cyan) shows a simulation where $\mathrm{S} 1$ is a dynamically switched resistance (between $0 \Omega$ and $100 \mu \Omega$ ), forming a two-switch pump. It can clearly be seen that a dynamically switched $\mathrm{S} 1$ is superior as it gives both a faster charging time and a higher final current than any of the fixed resistance single switch configurations.

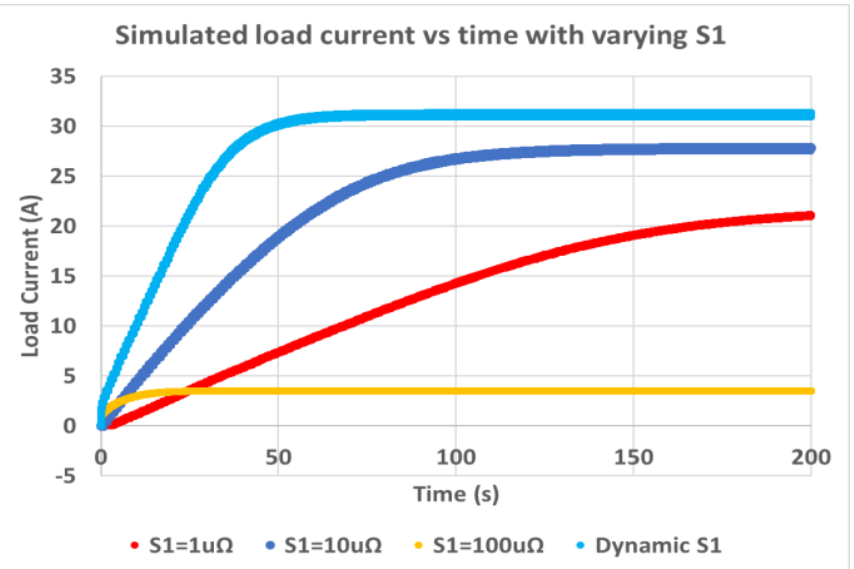

Figure 6: SPICE simulation of load current vs time in a TRFP employing different types of S1.

To verify the simulation seen in Figure 8, the same four situations were tested with the prototype. Agreement between simulation and prototype was very good. By making S1 a dynamic resistance, the power to the load can be maximized while keeping the loss low. If S1 is a static resistance, the load charging characteristic is dependent on the value of the S1 resistance value. Additionally, power is lost throughout the whole charging cycle, as opposed to just one half-cycle for the dynamically switched S1.

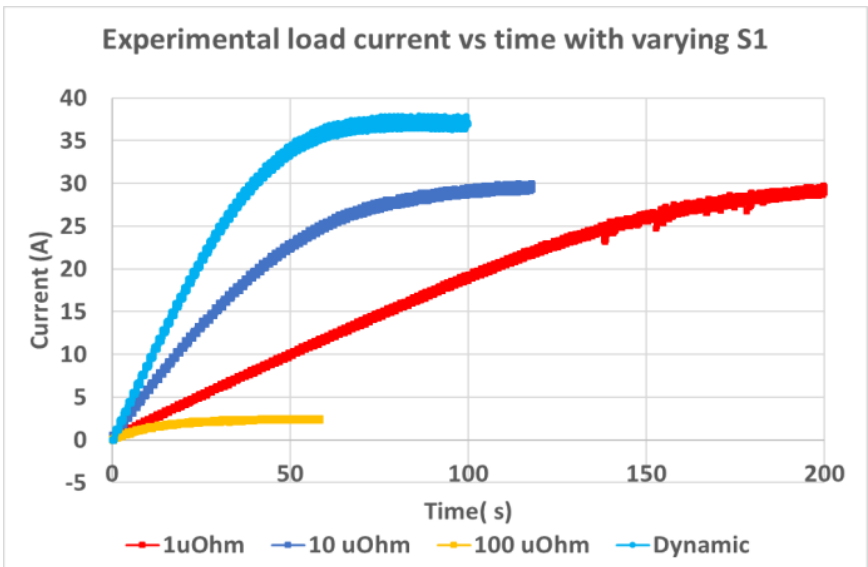

Figure 7: Experimental results of load current vs time in a TRFP employing different types of S1. Resistance values here are approximate and are calculated as mean voltage over mean current during the middle of switch off-time. In the experiment, the field strength applied to the HTS tape was reduced until the desired resistance was produced (for $10 \mu \Omega$ and $1 \mu \Omega$ ). 
As well as load current, it is useful to examine all the current, voltage and power waveforms for flux pumping circuits so they can be fully understood, designed for a specification and

Table 1: Results comparing simulation results to results measured from a prototype in the lab.

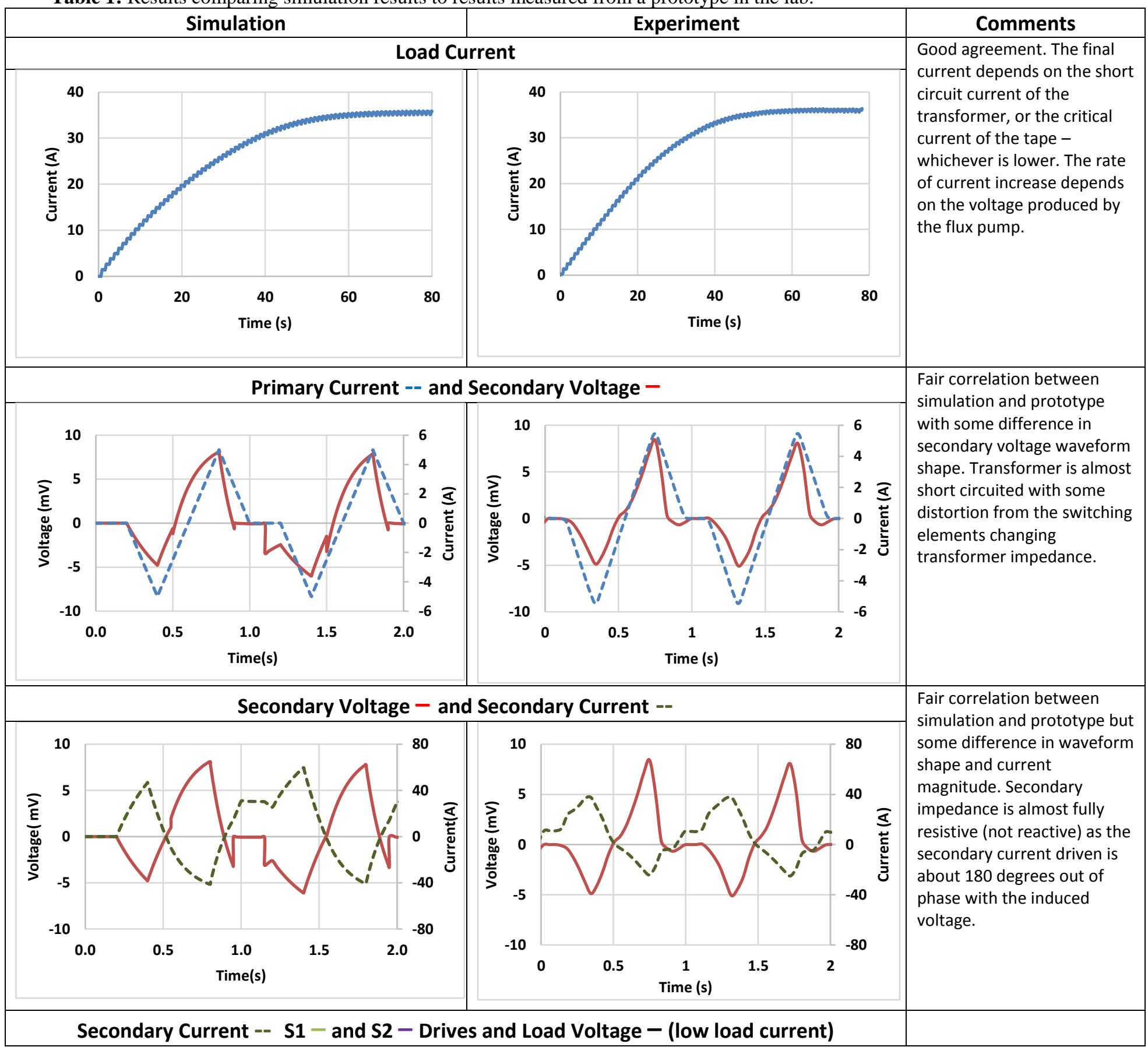

optimized. Table 1 compares the prototype waveforms with simulated waveforms for a TRFP employing a dynamically switched S1. 


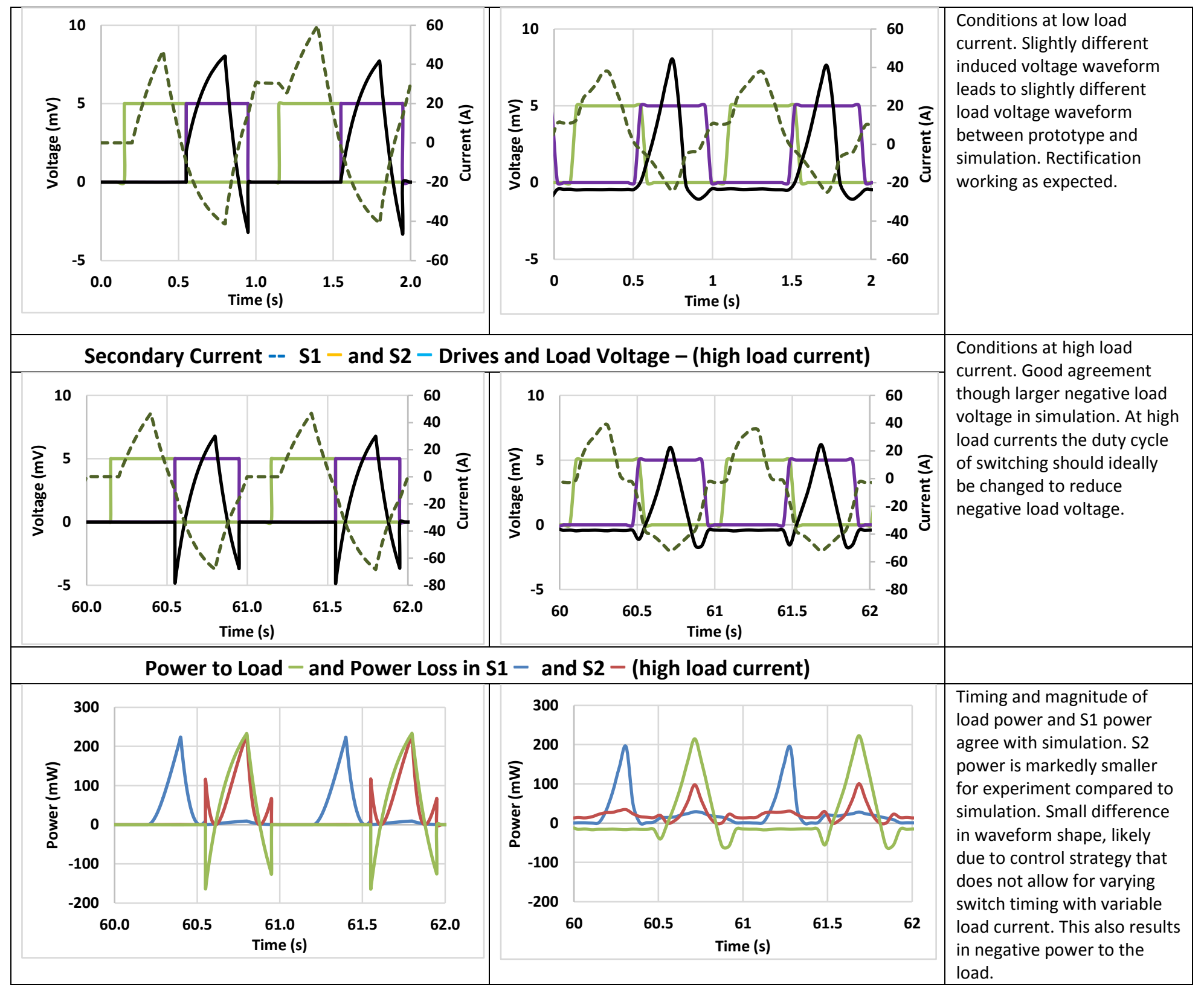

Examining these results, there is generally good qualitative agreement between simulated waveforms and measured waveforms. The agreement is inexact due to unaccounted for parasitic elements in the prototype and possibly measurement error and interference due to low voltage signals for the ADC used.

The first row in Table 1 shows the load current charging characteristic. There is good agreement between simulation and prototype both for final current and charging profile. The final current is largely determined by the critical current of the tape. This is simulated as a highly nonlinear resistance in series with the load.

The second row in Table 1 shows the voltage induced across the transformer secondary by the changing current in the primary. It must be noted that this transformer is not acting as a constant current or constant voltage transformer, rather somewhere in between and is dependent on the desired mode of operation. The operation of the transformer (constant voltage or constant current) is a very important consideration for future designs. A small variation in the impedance seen by the transformer can change the induced voltage waveform and therefore all other waveforms quite considerably. There is a slight discrepancy between simulation and prototype for this induced voltage signal however this does not affect the operation of the device significantly.

The third row in Table 1 shows the current driven in the transformer secondary by the induced voltage. It shows that current and voltage are almost in phase which means the transformer secondary sees a resistive load in this mode of operation. This phase relationship may change as the load charges depending on the design. There is a discrepancy between the magnitude of the transformer current waveforms. This may be due to unaccounted for parasitic impedance 
components in the transformer secondary. Again, this does not affect the operation significantly.

The fourth row in Table 1 shows the transformer secondary current, the two switch drives (S1 and S2) and the resulting load voltage at the beginning of pumping (low load current). When S2 is switched to high impedance, there is a voltage across the load. This is the voltage necessary for pumping a load magnet. It shows that there is some error in the timing of switching as some negative voltage is seen across the load. This is a known issue and is due to imperfect timing of switching S1 and S2 and can be improved on in future designs.

The fifth row in Table 1 shows the transformer secondary current, the two switch drives (S1 and S2) and the resulting load voltage at the end of pumping (high load current). There is now a larger negative voltage across the load. This shows that as the load current increases, the duty cycle of switching should be decreased.

The sixth row in Table 1 shows the power transferred to the load and the power losses in S1 and S2 at the end of charging. There is 'negative power' to the load due to the negative voltage across the load. This is well understood and can be resolved by using a correct control strategy. Other than this there is fairly good agreement between simulation and prototype with minor differences in waveform shape.

A full set of waveform results were also taken for a flux pump employing a static $\mathrm{S} 1$. These results will not be included for the sake of brevity, but they were as expected for both simulation and prototype.

\section{Discussion}

The results in section VI show that SPICE simulation and prototype measurements agree quite closely. The results demonstrate the advantage switching element S1 dynamically instead of employing a static $\mathrm{S} 1$. The results show in exact detail each step required in pumping flux into a load magnet, from the primary transformer current to the load current. The results show that the transformer is operating somewhere between a constant voltage transformer and constant current transformer. This is potentially not the ideal mode of operation and results in waveforms with high harmonic distortion. The results also show that incorrect timing of switching is limiting the operation of the flux pump. Simulation gives the designer a means identifying these issues and how to solve them.

Importantly this is the first time all voltage, current and power waveforms have been measured for any HTS flux pump. This is a crucial development as it gives a starting metric for further development. As this was an initial prototype with basic current and voltage measurements and a small-scale prototype, it is expected that the power levels can be dramatically improved on. For example, a simulation has been run that assumes perfect control, ignores core loss and eddy current loss from the AC electromagnets and employs switching elements $\mathrm{S} 1$ and S2 with off-state resistance of $100 \mathrm{~m} \Omega$. This simulation allows for a load current exceeding $8 \mathrm{kA}$ with a maximum power to the load exceeding $400 \mathrm{~W}$ at greater than $99 \%$ efficiency. This is an optimistic simulation, in particular the assumption of such high resistance switching elements, however it shows the potential performance of a correctly designed and optimized flux pump.
One major area for future work will be to design a control system for optimal operation of the pump. This will likely include feeding back signals such as load current into the Labview program that drives the transformer and switches, similar to the work detailed in Ref. [20]. It is expected that more parameters such as duty cycle and dead-time will also be varied based on feedback signals to enable lossless commutation of the load current between S1 and S2.

Another area of future work is to design HTS switching elements capable of developing higher off-state resistance and carrying more current. Efficiency is directly related to off-state resistance and load current is directly related to current handling ability of the switches. There needs to be a detailed analysis of the eddy current and magnetization loss in the HTS tape and its frequency and field strength dependence.

\section{Conclusions}

A half-bridge transformer-rectifier flux pump with two switching elements has been simulated in SPICE software and a prototype constructed. It has been demonstrated through simulation and prototype results that the two-switch half-bridge configuration is superior to a flux pump using just a single switching element. The results include all voltage, current and power waveforms for an HTS flux pump. There is generally good qualitative agreement between simulation and prototype. The simulation tool and measurement system provide designers of future flux pumping systems a means of understanding how to fully optimize their design and demonstrate the physical limits of this technology.

\section{Acknowledgments}

The Authors would like to acknowledge Mr John Grundy for his help in the laboratory. James Gawith would like to thank the Woolf Fisher Trust and Cambridge Trust for supporting his study in Cambridge.

\section{References}

[1] Y. Iwasa, 'HTS and NMR/MRI magnets: Unique features, opportunities, and challenges', Phys. C Supercond. Its Appl., vol. 445, no. Supplement C, pp. 1088-1094, Oct. 2006.

[2] 'To 20 Tesla and beyond: the high-temperature superconductors | CERN'. [Online]. Available:

https://home.cern/about/updates/2017/09/20-tesla-and-beyondhigh-temperature-superconductors. [Accessed: 04-Oct-2017].

[3] D. Uglietti, N. Bykovsky, R. Wesche, and P. Bruzzone, 'Development of HTS Conductors for Fusion Magnets', IEEE Trans. Appl. Supercond., vol. 25, no. 3, pp. 1-6, Jun. 2015. [4] P. J. Masson, G. V. Brown, D. S. Soban, and C. A. Luongo, 'HTS machines as enabling technology for allelectric airborne vehicles', Supercond. Sci. Technol., vol. 20, no. 8, p. 748, 2007.

[5] F. Bordry, A. Dupaquier, G. Fernqvist, H. E. Jorgensen, P. Madsen, and E. Steinmann, 'High Current, Low Voltage Power Converter [20kA, 6V] - LHC Converter Prototype', presented at the APS Meeting Abstracts, 1997, p. 5. 
[6] J. Geng et al., 'Origin of dc voltage in type II superconducting flux pumps: field, field rate of change, and current density dependence of resistivity', Feb. 2016.

[7] J. Geng and T. A. Coombs, 'Mechanism of a high- Tc superconducting flux pump: Using alternating magnetic field to trigger flux flow', Appl. Phys. Lett., vol. 107, no. 14, 2015. [8] C. W. Bumby, Z. Jiang, J. G. Storey, A. E. Pantoja, and R. A. Badcock, 'Anomalous open-circuit voltage from a highTc superconducting dynamo', Appl. Phys. Lett., vol. 108, no. 12, p. 122601, Mar. 2016.

[9] J. Geng, K. Matsuda, L. Fu, B. Shen, X. Zhang, and T. A. Coombs, 'Operational research on a high-Tc rectifier-type superconducting flux pump', Feb. 2016.

[10] C. W. Bumby, A. E. Pantoja, H. J. Sung, Z. Jiang, R. Kulkarni, and R. A. Badcock, 'Through-Wall Excitation of a Magnet Coil by an External-Rotor HTS Flux Pump', IEEE Trans. Appl. Supercond., vol. 26, no. 4, pp. 1-5, Jun. 2016. [11] R. A. Badcock et al., 'Impact of Magnet Geometry on Output of a Dynamo-Type HTS Flux Pump', IEEE Trans. Appl. Supercond., vol. 27, no. 4, 2017.

[12] C. W. Bumby et al., 'Frequency Dependent Behavior of a Dynamo-Type HTS Flux Pump', IEEE Trans. Appl.

Supercond., vol. 27, no. 4, 2017.

[13] L. J. M. van de Klundert and H. H. J. ten Kate, 'Fully superconducting rectifiers and fluxpumps Part 1: Realized methods for pumping flux', Cryogenics, vol. 21, no. 4, pp. 195-206, 1981.

[14] C. Hoffmann, D. Pooke, and A. D. Caplin, 'Flux Pump for HTS Magnets', IEEE Trans. Appl. Supercond., vol. 21, no. 3, pp. 1628-1631, Jun. 2011.

[15] Z. Bai, G. Yan, C. Wu, S. Ding, and C. Chen, 'A novel high temperature superconducting magnetic flux pump for MRI magnets', Cryogenics, vol. 50, no. 10, pp. 688-692, Oct. 2010.

[16] L. Fu, K. Matsuda, M. Baghdadi, and T. Coombs, 'Linear Flux Pump Device Applied to High Temperature

Superconducting (HTS) Magnets', IEEE Trans. Appl. Supercond., vol. 25, no. 3, pp. 1-4, Jun. 2015.

[17] S. Hahn, 'Linear flux pump: a potential alternative to energize superconducting magnet', Supercond. Sci. Technol., vol. 29, no. 7, p. 070502, Jul. 2016.

[18] J. Geng et al., 'Voltage-ampere characteristics of YBCO coated conductor under inhomogeneous oscillating magnetic field', Appl. Phys. Lett., vol. 108, no. 26, p. 262601, Jun. 2016.

[19] J. Geng and T. A. Coombs, 'An HTS flux pump operated by directly driving a superconductor into flux flow region in the E? J curve', Supercond. Sci. Technol., vol. 29, no. 9, p. 095004 , Sep. 2016.

[20] J. Geng et al., 'Feedback Control of a Rectifier Type HTS Flux Pump: Stabilizing Load Current With Minimized Losses', IEEE Trans. Appl. Supercond., vol. 27, no. 4, pp. 14, Jun. 2017.

[21] M. P. Oomen et al., 'HTS Flux Pump for Cryogen-Free HTS Magnets', IEEE Trans. Appiled Supercond., vol. 15, no. 2, pp. 1465-1468, Jun. 2005.

[22] J. Geng et al., 'HTS Persistent Current Switch Controlled by AC Magnetic Field', IEEE Trans. Appl. Supercond., vol. 26, no. 3, pp. 1-4, Apr. 2016.
[23] T. Ogasawara, K. Yasuköchi, S. Nose, and H. Sekizawa, 'Effective resistance of current-carrying superconducting wire in oscillating magnetic fields 1: Single core composite conductor', Cryogenics, vol. 16, no. 1, pp. 33-38, Jan. 1976. [24] V.V.Andrianov, V. B. Zenkevich, V.V.Kurguzov, V.V.Sychev, and (last) aooF. F.Ternovskii, 'Effective Resistance of an Imperfect Type II Superconductor in an Oscillating Magnetic Field', Sov. Phys. JEPT, vol. 31, no. 5, Nov. 1970.

[25] Z. Jiang, R. Toyomoto, N. Amemiya, X. Zhang, and C. W. Bumby, 'Dynamic resistance of a high- $\mathrm{T}$ c coated conductor wire in a perpendicular magnetic field at $77 \mathrm{~K}$ ', Supercond. Sci. Technol., vol. 30, no. 3, p. 03LT01, 2017. 